\title{
Reform des Europäischen Stabilitätsmechanismus - eine Einordnung
}

\author{
Ende November 2020 wurde eine Reform des Europäischen Stabilitätsmechanismus beschlossen, \\ die nicht unterschätzt werden sollte. Im Mittelpunkt steht die Einführung einer staatlichen \\ Letztabsicherung für den europäischen Bankenrettungsfonds ab 2022. Doch auch weitere \\ Änderungen sind relevant und werden in diesem Beitrag eingeordnet.
}

Ende November 2020 haben die Euro-Finanzminister eine relevante Reform des Euro-Rettungsschirms ESM (Europäischer Stabilitätsmechanismus) beschlossen. Vor allem die Einführung einer staatlichen Letztabsicherung für den europäischen Bankenrettungsfonds (Common Backstop) ab Anfang 2022 hat Schlagzeilen gemacht. Doch es gab auch weitere wichtige Entscheidungen der Eurogruppe, die in ihrer Bedeutung leicht unterschätzt werden können. Zudem wurde in Teilen der medialen Berichterstattung manches Detail nicht ganz richtig dargestellt. Um die Reformen aus politischer Sicht zu bewerten, ist es wichtig zu wissen, dass die ESM-Reformen im Grundsatz bereits Ende 2018 beschlossen, seitdem aber nicht umgesetzt wurden. Daher ist zu erörtern, was nun die Entscheidung ermöglicht hat, ob sich die Positionen wichtiger Akteure verschoben haben und warum das der Fall sein mag. Auch ist die Frage relevant, wie zwischen Nord- und Südeuropa die Balance von Geben (neue Hilfsmöglichkeiten) und Nehmen (mehr Regeldisziplin und weniger Moral Hazard) aussieht. ${ }^{1}$

\section{Backstop trotz mangelndem Risikoabbau}

Der Common Backstop ist als staatliche Letztsicherung (last resort) vorgesehen, falls bei einer Bankenkrise der von

(c) Der/die Autor:in(nen) 2021. Open Access: Dieser Artikel wird unter der Creative Commons Namensnennung 4.0 International Lizenz (https:// creativecommons.org/licenses/by/4.0/deed.de) veröffentlicht.

Open Access wird durch die ZBW - Leibniz-Informationszentrum Wirtschaft gefördert.

1 Die folgenden Ausführungen basieren auf den Dokumenten der Eurogruppe (2020) sowie des ESM (2020a, b, c, d).

Jürgen Matthes ist Senior Economist und Leiter des Kompetenzfelds „Internationale Wirtschaftsordnung und Konjunktur" im Institut der deutschen Wirtschaft. den europäischen Banken gefültte Bankenrettungsfonds nicht ausreicht. Anders als teilweise in der Berichterstattung dargestellt, war der Backstop schon im Zuge der Euro-Schuldenkrise im Grundsatz beschlossen worden. Demnach soll die damit verbundene Kreditlinie des ESM spätestens Anfang 2024 in Kraft treten. Viele Eurostaaten wollten aber eine frühere Einführung. Doch Deutschland und einige andere Länder hatten dies hinausgezögert mit dem berechtigten Hinweis, dass nach der Euro-Schuldenkrise in erster Linie einige südeuropäische Banken noch zu viele Altlasten in den Bilanzen hatten. Neben notleidenden Krediten spielte dabei auch die mangelnde Vorsorge für zukünftige Krisen eine Rolle, insbesondere in Form von bailin-fähigem Bankenkapital, um im Krisenfall Eigentümer und Gläubiger an Verlusten beteiligen zu können statt die Steuerzahlenden. Dahinter stand die Sorge, dass nordeuropäischen Steuerzahlenden neue Haftungsrisiken aufgebürdet würden für Versäumnisse in Südeuropa. Daher wurde das Vorziehen des Backstop an einen ausreichenden Abbau der Risiken und an Mindestanforderungen für bailin-fähiges Bankenkapital (MREL - Minimum Requirement for Own Funds and Eligible Liabilities) gebunden, vor allem mit Blick auf den südeuropäischen Bankensektor. Zudem hatte die Bundesregierung gefordert, dass die Insolvenzregeln für Unternehmen stärker vereinheitlicht werden und die Banken weniger Anleihen des eigenen Staats in ihren Bilanzen halten sollten, damit Schuldenprobleme der Staaten nicht die Banken mit in die Krise reißen.

Die Einführung des Common Backstop wurde wohl vor allem vor dem Hintergrund der Corona-Krise beschlossen, und das, obwohl der Risikoabbau im Bankensektor nicht in allen Bereichen ausreichend war. Auch die Bundesregierung hat damit ihre Position verschoben und reagiert auf die veränderte Lage. Ähnlich wie beim europäischen Corona-Aufbaupaket soll demonstriert werden, dass Europa handlungsfähig ist und die Krise im Griff hat. Tatsächlich ist die staatliche Absicherung des Bankenrettungsfonds als vertrauensbildendes Signal wichtig, denn im Zuge der Krise werden europäische Banken vor allem durch Kreditausfälle stärker unter Druck kommen. Damit 
wird sich auch der trendmäßige Abbau der Bankenrisiken wieder umkehren.

Ein genauerer Blick in den vorgelegten Risikobericht (European Commission Services et al., 2020) zeigt, dass der Fortschritt beim Risikoabbau - anders als von der Eurogruppe behauptet - nicht wirklich zufriedenstellend ist. Zwar hat sich das Volumen der notleidenden Kredite im europäischen Bankensektor von rund 1.000 Mrd. Euro im vierten Quartal 2014 auf rund 500 Mrd. Euro im zweiten Quartal 2020 halbiert, auch weil die Bankenaufsicht hier erheblichen Druck gemacht hat. Doch die Risiken bleiben trotz nennenswerter Fortschritte noch zu sehr auf Problemfälle konzentriert, was bei einer neuerlichen Krise zu Bankenpleiten und damit über systemische Ansteckungseffekte zur Gefahr für das europäische Bankensystem werden kann. Für den Risikoabbau bei notleidenden Krediten (inklusive Vorauszahlungen, ohne Ausfallvorsorge) hatte die Eurogruppe schon Ende 2018 einen Zielwert von 5\% an allen Krediten für die relevanten Banken vorgegeben. Bei dem schlechtesten Viertel der Banken lag der gewichtete Durchschnitt des Anteils notleidender Kredite im zweiten Quartal 2020 jedoch noch bei 9,2\% (damit aber immerhin rund zwei Drittel niedriger als Ende 2014). Die Problembanken sind in einigen südeuropäischen Ländern konzentriert. So lag der gewichtete Anteil notleidender Kredite zuletzt in Griechenland bei $30 \%$, in Zypern bei über 13\%, in Portugal bei 6,5\% und in Italien bei 6,1\%. Deutschland weist im zweiten Quartal 2020 einen Wert von 1,2\% auf.

Gravierender sind die insgesamt mangelnden Fortschritte bei MREL. Die Mindestanforderungen an das bail-in-fähige Bankenkapital sollen bis 2024 kontinuierlich aufgebaut werden. Doch schon Ende 2019 bestand noch eine Lücke von 74 Mrd. Euro. Diese hat sich bis zum zweiten Quartal 2020 auf rund 147 Mrd. Euro fast verdoppelt. Relativ zum gesamten aggregierten Bankenrisiko sind das rund $2 \%-$ gegenüber $1 \%$ im vierten Quartal 2019. Die Verschlechterung liegt vor allem daran, dass die Corona-Krise den Aufbau der Puffer erschwert hat und der Zielwert 2021 wie vorgesehen gestiegen ist. Besonders hohe MREL-Lücken finden sich in Griechenland, wo knapp die Hälfte der Mindestkapitalanforderungen noch nicht erreicht ist, sowie in Zypern und Portugal, wo der fehlende Anteil bei knapp einem Drittel von MREL liegt. Deutschland hingegen - ebenso wie Italien - hat die MREL-Anforderungen erfüllt.

So bemerkenswert die veränderte Position Deutschlands beim Backstop ist, so fragwürdig ist die hierzulande teilweise zu vernehmende Kritik, dieser Schritt würde die Haftungsrisiken für die nordeuropäischen Steuerzahlenden deutlich erhöhen. Denn zum einen können auch nordeuropäische Banken Probleme bekommen. Und zum anderen wird mit der Einführung des Backstop mit einem Volumen von maximal 68 Mrd. Euro ein anderes (bislang ungenutztes) Instrument des ESM in einem Umfang von maximal 60 Mrd. Euro auslaufen. Es handelt sich um die Möglichkeit des ESM, Krisenbanken direkt zu rekapitalisieren. Damit wären deutlich höhere Verlustrisiken verbunden gewesen, weil der ESM Miteigentümer geworden wäre. Beim Backstop geht es zwar um ein etwas höheres Volumen, dafür ist vorgesehen, dass der Bankenrettungsfonds in maximal fünf Jahren die Hilfskredite des ESM zurückzahlt, finanziert durch die europäischen Banken. Die Behauptung, durch die Reform sollten europäische Steuerzahlende für Pleitebanken haften, stimmt daher in dieser pauschalen Form nicht.

\section{Zugang zu vorsorglicher Kreditlinie erleichtert}

Als weiteres wichtiges Element der ESM-Reform hat die Eurogruppe beschlossen, den Zugang zu einer der bestehenden vorsorglichen Kreditlinien zu erleichtern. Anders als zuweilen von Medien dargestellt, wird damit kein neues Instrument geschaffen.

Es gab bisher zwei vorsorgliche Kreditlinien, die PCCL (Precautionary Conditioned Credit Line) und die ECCL (Enhanced Conditions Credit Line). ${ }^{2}$ Wenn ein Land die jeweiligen Bedingungen erfüllt, stellt der ESM bei beiden eine Kreditlinie zur Verfügung, die bei Bedarf gezogen werden kann, oder gibt die Zusage, Staatsanleihen des betreffenden Staats am Primärmarkt zu kaufen (ESM, 2020e). PCCL und ECCL werden zunächst für ein Jahr vergeben mit der zweimaligen Möglichkeit einer Verlängerung um jeweils sechs Monate. Zudem gibt es eine Ex-ante-Konditionalität, also eine Vorqualifizierung. Bei der PCCL muss der Eurostaat Kriterien erfüllen, wie die Vorgaben der fiskalischen und makroökonomischen Überwachung und insbesondere Regeln des Stabilitäts- und Wachstumspakts. Bei der ECCL können bei einzelnen dieser Vorgaben Abstriche gemacht werden, aber generell muss die ökonomische und finanzielle Situation hinreichend gut sein. Als Gegenbuchung für diese lockereren Ex-ante-Bedingungen sind die Ex-post-Bedingungen in Form von Reformauflagen bei der ECCL umfangreicher als bei der PCCL (allerdings bei beiden geringer als bei einem dreijährigen Vollprogramm der ESM). All diese Bedingungen werden in einem sogenannten Memorandum of Understanding (MoU), einer Art Reformvertrag festgelegt. Neu ist bei der PCCL, dass es in Zukunft kein MoU mehr geben wird. Es reicht aus, dass der betreffende Eurostaat in einem Absichtsbrief (Letter of Intent) festlegt, mit welcher Politikausrichtung er die Vorbedingungen während der Laufzeit einhalten will. Die Europäische Kommission prüft diese Absichtserklärung und gibt

2 Auf Basis der ECCL wurde zudem in der Corona-Krise ein nur krisenbezogenes Pandemic Credit Support geschaffen, mit der aber nur Ausgaben mit Pandemiebezug finanziert werden können. 
gegebenenfalls grünes Licht. Ob während der Laufzeit der Kreditlinie alle Vorbedingungen erfüllt bleiben, prüft halbjährlich der ESM; nach dem Ziehen der Kreditlinie nimmt er unmittelbar eine solche Prüfung vor. Wird eine Verletzung festgestellt, endet die Kreditlinie, außer das Direktorium ist einstimmig dagegen. Sofern bereits Kredite gezogen wurden, wird darauf in der Regel die Zinsmarge erhöht.

Hiermit wird der Zugang zum ESM (unter den besagten Voraussetzungen) deutlich erleichtert. Weil ein MoU mit Reformvorgaben einhergeht und das wie bei den ESM-Vollprogrammen während der Euro-Schuldenkrise als ein Verlust an nationaler Souveränität wahrgenommen wurde, ist der ESM in Südeuropa unbeliebt und wird so lange umgangen wie möglich. Durch den Verzicht auf ein MoU wird der ESM ein gutes Stück attraktiver. Das ist ein bedeutsamer Schritt, weil der ESM eine wichtige Säule in der Architektur der Europäischen Währungsunion ist. Zudem wird mit der Ex-ante-Konditionalität der Anreiz weiter gefördert, die EWU-Regeln einzuhalten. Wieso sind diese Erleichterungen beim Zugang zu ESM-Krediten möglich geworden, spiegeln sie doch grundsätzlich eher die Interessen der südeuropäischen Staaten wider? Hier reicht der Verweis auf die aktuelle Krise nicht aus. Der Blick ist auch dahin zu öffnen, dass die nordeuropäischen Staaten im Ausgleich für die PCCL-Veränderung (und die frühzeitige Backstop-Einführung) auch zwei politische Gegenbuchungen durchgesetzt haben, auf die sich die südeuropäischen Länder nach langem Zögern nun eingelassen haben: Erleichterungen bei Umschuldungen von Staatsschulden und eine größere Rolle des ESM in Relation zur Europäischen Kommission.

\section{Erleichterung von Umschuldungen}

Wenn Staatsschulden nicht mehr tragfähig sind, Zahlungsunfähigkeit und Staatsbankrott drohen und auch ein ESM-Kredit nicht mehr helfen sollte, würde als Ultima Ratio eine Umschuldung nötig. Dabei verhandelt der betroffene Staat mit seinen Gläubiger über einen Forderungsverzicht, um die Schulden wieder tragfähig zu machen. Dabei kann ein Problem auftreten: Je nach den Bestimmungen im Kleingedruckten der Staatsanleihen kann es dazu kommen, dass einzelne Gläubiger (Hold-outs) ihre Zustimmung verweigern und ihre Forderungen sogar vollständig einklagen. Damit agieren sie nicht nur auf Kosten des betroffenen Staates, sondern auch zulasten der anderen Gläubiger, die prozentual auf einen höheren Anteil ihrer Forderungen verzichten müssten, um Staatsschulden wieder tragfähig zu machen (Busch und Matthes, 2015).

Um dieses Risiko zu vermindern, haben die Eurostaaten ab Anfang 2013 Abstimmungsregeln (Collective Action Clauses - CACs) in das Kleingedruckte ihrer neuen Staatsanleihen aufgenommen. Sie sehen vor, dass eine qualifizierte Mehrheit der Gläubiger die verbleibende Minderheit (und damit auch potenzielle Hold-out-Gläubiger) dazu zwingen kann, an einer Umschuldung teilzunehmen. Allerdings ist diese Abstimmung sowohl auf der Ebene aller Staatsanleihen nötig als auch auf der Ebene einzelner Emissionsserien. Die Abstimmung auf Serienebene bietet Hold-out-Gläubigern die Möglichkeit, mit einem überschaubaren Anlagevolumen eine Blockademinderheit an Staatsanleihen aufzukaufen und sich so der Umschuldung zu entziehen.

Die neue ESM-Reform sieht vor, dass künftig nur noch eine Abstimmung für alle Serien nötig ist und keine weiteren auf Serienebene. Dazu sollen ab Anfang 2022 sogenannte Single-Limb CACs eingeführt werden für neue Staatsanleihen der Euroländer. Darüber hinaus wird dem ESM die Möglichkeit gegeben, bei Umschuldungsverhandlungen als informeller und verschwiegener Moderator aufzutreten und diese so idealerweise zu erleichtern. Der betreffende Staat kann dies freiwillig und vorübergehend anfordern und hat dazu durchaus einen Anreiz, weil der ESM guten Kontakt zu Investoren hat. Mit diesen Reformschritten werden Umschuldungen tendenziell erleichtert. Aus diesem Grund war vor allem Italien lange gegen diese Reform (Reuters, 2019). Man fürchtete, dass dadurch die Risikoaufschläge auf die Zinsen der eigenen Staatsanleihen steigen könnten. Es kann nur spekuliert werden, warum die italienische Regierung nun zugestimmt hat. Eventuell ist es die Einsicht, dass auch die Einführung der CACs 2013 zu keinen größeren Zinsveränderungen geführt hat, obwohl damals die Euro-Schuldenkrise noch schwelte. Möglicherweise stimmte sie zu, weil sie davon ausgeht, dass die Präsenz der Europäischen Zentralbank in den Staatsanleihemärkten auch bei der Einführung der neuen CACs 2022 einen größeren Anstieg der Risikoprämien verhindert. Vielleicht haben aber auch die Erleichterungen bei der PCCL und vor allem die großzügigen Regelungen des EU-Aufbaufonds, von dem Italien besonders profitiert, die politische Blockade gelöst und die italienische Regierung zu mehr europäischer Kooperationsbereitschaft veranlasst.

Trotz der Einführung von Single-Limb-CACs bleiben Umschuldungen unwahrscheinlich. Denn gerade auch in einigen südeuropäischen Staaten halten die Banken relativ viele Staatsanleihen in ihren Bilanzen. Als Gläubiger müssten sie auf einen Teil dieser Forderungen verzichten und damit erhebliche Bilanzverluste verkraften, was Bankenpleiten auslösen könnte. Wegen dieser Ansteckungsgefahr wird die Eurogruppe Staatsbankrotte um womöglich jeden Preis zu verhindern suchen und dazu gegebenenfalls die Interventionen der EZB noch stärker nutzen wollen. Daher sollte die Bundesregierung ihre frühere Forderung erneuern und mit Nachdruck für eine Begrenzung der Staatsanleihen in den Bankbilanzen eintreten. 


\section{Größere Rolle des ESM}

Der ESM erhält eine größere Rolle bei Hilfs- und Reformprogrammen im Vergleich zur Europäischen Kommission. Das ist deshalb von Bedeutung, weil der ESM stärker sachlich-technisch und damit weniger politisch agieren dürfte als die Europäische Kommission. Da Reformprogramme unbeliebt sind, ist die Gefahr groß, dass sich die Europäische Kommission, wenn sie allein agieren würde, auf zu große Kompromisse einlassen würde, was wirtschaftlich nicht sachdienlich sein könnte. Aus diesem Grund waren auch bislang schon die EZB und, wenn möglich, der Internationale Währungsfonds (IWF) bei wichtigen Entscheidungen eingebunden. Die Reform sieht vor, dass der ESM - neben der Europäischen Kommission in Verbindung mit der EZB - die Aufgaben bekommt, Reformauflagen (Konditionalität) mit dem betreffenden Mitgliedstaat zu verhandeln (weiterhin ohne den IWF) und später die Einhaltung der Reformkonditionalität zu überwachen (wenn möglich mit dem IWF).

Darüber hinaus erhält der ESM eine wichtige Rolle bei der Prüfung der Grundvoraussetzungen für ESM-Hilfsprogramme. Neben der immer schon vorgeschalteten Schuldentragfähigkeitsanalyse wird durch die Reform als weitere Voraussetzung die Prüfung eingeführt, ob der Krisenstaat den ESM-Kredit zurückzahlen können wird. Zudem sieht die Reform bei der Bewertung beider Aspekte nun explizit einen Ermessensspielraum vor. Die nötigen Beurteilungen obliegen in Zukunft dem ESM (genauer der geschäftsführenden Direktion) zusätzlich und zusammen mit den bisherigen Institutionen: der Europäischen Kommission in Verbindung mit der EZB und, wenn möglich, dem IWF. Der ESM kann und soll dabei seine Finanzmarktexpertise einbringen. Wenn diese Institutionen nicht zu einer gemeinsamen Lösung finden, beurteilt die Europäische Kommission allein fiskalische und makroökonomische Aspekte und der ESM allein die Rückzahlungsfähigkeit. Damit wird der ESM etwas mehr zu einem Europäischen Währungsfonds (EWF). Was genau einen EWF ausmacht, ist zwar nicht klar definiert (Matthes, 2017), aber wenn man den IWF zum Vorbild nimmt, dann bekommt der ESM eine ähnliche Rolle bei Hilfs- und Reformprogrammen. Bei der makroökonomischen Länderanalyse ist er nicht so breit und tief aufgestellt, dafür hat er durch die Emission eigener Anleihen mehr praxisbezogenes Know-how mit Blick auf den Finanzmarkt und seine Akteure.
Fazit

Insgesamt ermöglicht die ESM-Reform zwar keine grundlegende Neuaufstellung. Doch mit der Backstop-Einführung wird ein wichtiges Signal der Handlungsfähigkeit gesendet, ohne die Risiken für die Steuerzahlenden nennenswert zu erhöhen. Zudem wird mit der PCCL-Erleichterung und der erweiterten Rolle des ESM an weiteren Stellschrauben in die richtige Richtung gedreht. Damit wird der ESM handlungsfähiger und Umschuldung etwas erleichtert. Bemerkenswert ist, dass sich vor dem Hintergrund der Corona-Krise die politischen Blockaden gelöst haben. So konnte ein Reformpaket umgesetzt werden, das eine angemessene Balance zwischen den Interessen von Nord und Süd im Euroraum hat. Es bleibt abzuwarten, ob sich die stärkere Bereitschaft zur Zusammenarbeit gerade auch zwischen Italien, Frankreich und Deutschland angesichts der Krise und der umfangreichen Krisenhilfen auch in die Zukunft fortschreiben lässt.

\section{Literatur}

Busch, B. und J. Matthes (2015), Regeln für Staatsinsolvenzen im Euroraum, IW-Analyse, Nr. 104

ESM (2020a), Agreement Amending the Treaty Establishing the European Stability Mechanism, https://www.consilium.europa.eu/media/47294/ sn04244-en19.pdf (20. Dezember 2020).

ESM (2020b), ESM Treaty Reform - Explainer, https://www.esm.europa.eu/ about-esm/esm-treaty-reform-explainer\#ui-id-1 (20. Dezember 2020).

ESM (2020c), ESM-Reform, https://www.esm.europa.eu/about-esm/esmreform u.a. (20. Dezember 2020).

ESM (2020d), Treaty Establishing the European Stability Mechanism, https://www.esm.europa.eu/sites/default/files/20150203_-_esm_treaty_-_en.pdf (20. Dezember 2020).

ESM (2020e), European Stability Mechanism Guideline on Precautionary Financial Assistance, https://www.esm.europa.eu/sites/default/files/ esm_guideline_on_precautionary_financial_assistance.pdf (20. Dezember 2020).

European Commission Services, ECB und SRB (2020), Monitoring Report on Risk Reduction Indicators, November, Brüssel, https://www.consilium. europa.eu/media/46978/joint-risk-reduction-monitoring-report-to-eg_ november-2020_for-publication.pdf (20. Dezember 2020).

Eurogruppe (2020), Statement of the Eurogroup in inclusive format on the ESM reform and the early introduction of the backstop to the Single Resolution Fund, Press Release, 30. November, https://www.consilium. europa.eu/en/press/press-releases/2020/11/30/statement-of-the-eurogroup-in-inclusive-format-on-the-esm-reform-and-the-early-introduction-of-the-backstop-to-the-single-resolution-fund/ (20. Dezember 2020).

Matthes, J. (2017), A European Monetary Fund - Considerations of Design, Politics and a Preliminary Evaluation, CESifo-Forum, 18(3), 16-20.

Reuters (2019), Italian coalition parties seek changes to ESM debt restructuring terms, 9. Dezember, https://www.reuters.com/article/italy-esmidUSS8N28E0BD (20. Dezember 2020).

Title: Reform of the European Stability Mechanism - A Classification

Abstract: At the end of November 2020, a reform of the European Stability Mechanism was adopted that should not be underestimated. The article focuses on the introduction of a common fiscal backstop for the European Single Resolution Fund from 2022, as well as other relevant changes. Additionally, the article provides a political evaluation.

JEL Classification: H12, G01, 052 